\title{
The quest for affordable artemisinin
}

\author{
"Interestingly, the traditional approach to small-molecule manufacture - organic synthesis - has been \\ largely ignored in the pursuit of low-cost artemisinin."
}

Keywords: artemisinin $\approx$ drug discovery $\approx$ malaria $\approx$ synthesis $\approx$ total synthesis

Malaria, caused by a parasitic infection of the red blood cells and liver, represents one of the most impactful diseases of the last 100 years. Driving tactical decisions of most major Eurasian wars of the 20th century, malaria has also destroyed peacetime economies. Fortunately, untreated malaria has a low rate of mortality, generally below $1 \%$. However, the mortality rate is highest in young children, resulting in greater than $90 \%$ of the approximately one million deaths per annum affecting the 5-year-old or younger demographic. Furthermore, one in four adults in endemic countries are unable to work at any given time due to infection, taking an enormous economic toll on these populations. Particularly hard hit are those countries of sub-Saharan Africa where malaria is directly responsible for more than US $\$ 40$ billion/year in lost productivity, representing a significant reduction of gross domestic product for these nations. Consequently, in terms of transformative goals for the betterment of sub-Saharan Africa, the suppression or eradication of malaria-causing Plasmodium infections ranks close to improved water infrastructure and better farming techniques.

Quinine has been employed in the fight against malaria since the 17 th century, and continues to serve a need even today among malaria-stricken pregnant women [1]. Generally usurped in the 1940s by drugs with fewer deleterious side effects, quinine is still used by rural populations due to its broad availability and low cost. While chloroquine, introduced clinically in the late 1940s, has few side effects and could be used prophylactically, the emergence of chloroquine-resistant Plasmodium strains in the 1950s heralded the decline of this miracle drug and ignited a multinational race to discover more effective antimalarials [2]. Piperaquine followed in the 1960s and was used widely in China and Indo-China through the 1980 s, at which time resistance arose and better drugs penetrated the market. Mefloquine was developed by the US
Army in the 1970s but has met limited deployment due to reports of serious neuropsychiatric problems [3,4], some of which may be mitigated through the use of a single-enantiomer formulation $[5,6]$. Other drugs, such as lumefantrine, pyronaridine, primaquine and pyrimethamine, have also been used in various combinations starting in the 1970s, but many have limited efficacy as a stand-alone medications or have led to resistant Plasmodium strains [2].

The natural product artemisinin was isolated from the sweet wormwood plant, Artemesia annua, in 1972 during a program started by the Chinese army in the 1960s, code-named Project 523 [7]. This unique compound, the first natural product containing an endoperoxide, demonstrated rapid clearance of various Plasmodium species in mice. A clinical trial of 2000 patients confirmed the remarkable drop in parasite count in humans, but artemisinin also demonstrated poor pharmacokinetics with incomplete bioavailability and a 2-5 h half-life [8]. With the rapid clearance of the parasite, patients often felt better within $24 \mathrm{~h}$, which led to discontinuation of the 14-day treatment [9] and the return of the disease when the original, uncleared parasites rebound. This prompted the investigation of other, more persistent antimalarial drugs in combination with artemisinin or artemisinin derivatives, known as artemisinin-based combination therapies (ACTs). Currently, artemether/lumefantrine and artesunate/amodiaquine combination therapies have been widely adopted for the treatment of uncomplicated malaria across sub-Saharan Africa [10]. While better than artemisinin alone, these ACTs still require a 3-day treatment regimen, which is long enough to limit compliance among many endemic populations. In addition, ACTs are significantly more expensive than artemisinin alone, thereby suppressing adoption by rural populations and leading to a deluge of counterfeit drugs in a growing number of urban pharmacies. While the ideal solution would be a Plasmodium vaccine, or some other long-term

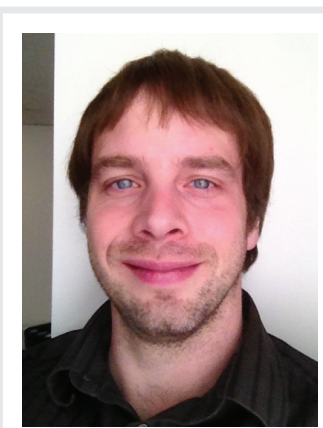

Silas P Cook

Indiana University, 800 E. Kirkwood Avenue, Bloomington, IN, 47405, USA

E-mail: sicook@indiana.edu 
prophylactic cure, recent setbacks in parasite vaccine research have made the need for an affordable, single-dose malaria cure as important as ever [11].

"While the ideal solution would be a
Plasmodium vaccine, or some other long-term
prophylactic cure, recent setbacks in parasite
vaccine research have made the need for an
affordable, single-dose malaria cure as
important as ever."

After many years of research into the mechanism of action of artemisinin, scientists remain at odds over the precise details. When Plasmodium infects red blood cells, the parasite consumes hemoglobin and polymerizes heme into hemozoin to avoid the cell toxicity of free heme. While the target of artemisinin is generally believed to be heme, it is not the typical 'lock-and-key' paradigm of protein-small-molecule recognition, but rather a reaction between heme and artemisinin that sets in motion a cascade of events unfavorable to the parasite. It is clear that reductive activation of the endoperoxide in artemisinin by the iron(II) center of heme triggers the generation of highly reactive radical species. However, the degree to which reactive oxygen radicals, alkylation of heme by C-centered radicals, or simple disruption of parasitic redox homeostasis account for parasite death is unclear $[12,13]$.

One approach toward a single-dose malaria cure has been the synthesis of small molecules with improved pharmacokinetics and efficacy based on the 1,2,4-trioxane substructure, or endoperoxide, responsible for the antimalarial activity of artemisinin. Synthetic trioxanes also alkylate heme and their rate of alkylation correlates well with their antimalarial efficacy [12]. This medicinal chemistry approach, pioneered by luminaries such as Professor Posner and Professor Vennerstrom among others, has produced a number of lead compounds with greater efficacy than artemisinin itself [14]. Since cost, distribution and compliance are the major hurdles in the fight against malaria, the time and money required to take drug candidates through clinical trials limits the near-term impact of this approach [15]. While these programs will likely produce the next generation of excellent antimalarials, only time will tell whether these can be delivered to those who need them the most at the rock-bottom pricing required to make a difference.
Vaccine research or new chemical entity (NCE) development coupled to clinical trials, while necessary, take significant time and money. Meanwhile, current therapies still suffer from issues of cost, compliance and distribution. A bridge to the vaccine or prophylactic cure will likely require broader use of artemisinin and its currently approved analogs. By combining these compounds with other antimalarials and/ or compounds that facilitate the oxidative damage of parasites, it may be possible to achieve a single-dose regimen. Furthermore, discovering improved formulations or delivery vehicles that enhance the bioavailability or blood half-life of artemisinin could be another avenue to fewer treatments and reduced costs. If such technologies could be identified, the path to approval and deployment would be vastly accelerated relative to clinical trials needed for a NCE. Consequently, access to lower-cost artemisinin and its derivatives will be of central importance in the near term.

\section{"While these programs will likely produce the next generation of excellent antimalarials, only time will tell whether these can be delivered to those who need them the most at the rock-bottom pricing required to make a difference."}

During the last 10 years, the quest to reduce the cost of artemisinin has been mostly confined to biological strategies. For example, Artemesia plants with improved growth traits and higher artemisinin yields would facilitate current methods of artemisinin production. However, the selective breeding of Artemesia, or even fast-track breeding based on the genetic map of the plant [16], will require many years of dedicated, meticulous farming since Artemesia requires at least 6 months to reach maturity. Another example, an early foray into synthetic biology, has resulted in astonishing microbial systems capable of producing chemical precursors to artemisinin [17]. While obtaining commercially viable titers of these chemicals has been challenging [18], this approach will likely provide the world with consistent artemisinin supplies, thereby removing the recent price fluctuations that stem from typical issues of commercial farming. Unfortunately, the microbially produced semisynthetic artemisinin retains approximately the same price as natural artemisinin. 
Interestingly, the traditional approach to small-molecule manufacture - organic synthesis - has been largely ignored in the pursuit of low-cost artemisinin. Early total syntheses of artemisinin in the 1980s delineated interesting, high-value strategies and provided the foundations for modern approaches to endoperoxide synthesis. The need for a low-cost synthesis of artemisinin, however, was unforeseen at the time. Once the shortages and severe price swings inherent to commercial farming became apparent in the 2000s, the merit of new, cost-conscious synthetic strategies for artemisinin became apparent [19]. We recently provided a blueprint for the low-cost synthesis of artemisinin from cheap, widely available commodity chemicals [20]. Our step-economical approach provides a proof-of-concept that this relatively complex natural product can be reduced to practice with straightforward chemistry. With the cost of raw materials at approximately $\$ 0.50-0.70 / \mathrm{g}$ of artemisinin, the synthesis provides a solid starting point for investigation by process chemists. More importantly, this versatile synthetic strategy can potentially be used for the production of other approved artemisinin derivatives. This could be done directly from a common intermediate, in the same number of steps, without the need to synthesize artemisinin [101].

The free-market societies of the world will continue to support drug development in therapeutic

\section{References}

1 Chico RM, Chandramohan D. quinine for the treatment of malaria in pregnancy. Lancet Infect. Dis. 10(3), 140-141 (2010).

2 Dandapani S, Comer E, Duvall JR, Munoz B. Hits, leads and drugs against malaria through diversity-oriented synthesis. Future Med. Chem. 4(18), 2279-2294 (2012).

3 Croft AM, World MJ. Neuropsychiatric reactions with mefloquine chemoprophylaxis. Lancet 347(8997), 326 (1996).

4 Gullahorn GM, Bohman HR, Wallace MR. Anaesthesia emergence delirium after mefloquine prophylaxis. Lancet 341(8845), 632 (1993).

5 Knight JD, Sauer SJ, Coltart DM. Asymmetric total synthesis of the antimalarial drug (+)-mefloquine hydrochloride via chiral $N$-amino cyclic carbamate hydrazones. Org. Lett. 13(12), 3118-3121 (2011).

6 Schmidt M, Sun H, Rogne P et al. Determining the absolute configuration of (+)-mefloquine $\mathrm{HCl}$, the side-effect-reducing areas that offer the greatest potential for financial return. However, the technologies that will save the greatest number of lives and bring billions out of poverty will likely be simple, elegant solutions to long-standing problems. In this regard, organic chemistry is ideally positioned to leverage the creativity of the next generation of chemists to redefine the meaning of 'elegance' in the context of scientific solutions. More often than not, future elegant syntheses will consist of practical, scalable reactions that rapidly build complexity and utilize environmentally friendly reagents and catalysts. By delivering these solutions for pennies a treatment, organic chemists have the potential to make transformative changes in places such as Africa. While saving lives is a noble endeavor in its own right, healing disease-stricken economies will allow struggling countries to forge their own paths to independence, prosperity and good health.

\section{Financial \& competing interests disclosure}

The author has no relevant affiliations or financial involvement with any organization or entity with a financial interest in or financial conflict with the subject matter or materials discussed in the manuscript. This includes employment, consultancies, honoraria, stock ownership or options, expert testimony, grants or patents received or pending, or royalties.

No writing assistance was utilized in the production of this manuscript. enantiomer of the antimalaria drug Lariam. J. Am. Chem. Soc. 134(6), 3080-3083 (2012).

7 Liu J-M, Ni M-Y, Fan J-F et al. Structure and reaction of arteannuin. Hua. Xue. 37(2), 129-143 (1979).

8 De Vries PJ, Dien TK. Clinical pharmacology and therapeutic potential of artemisinin and its derivatives in the treatment of malaria. Drugs 52(6), 818-836 (1996).

9 Kumar N, Sharma M, Rawat DS. Medicinal chemistry perspectives of trioxanes and tetraoxanes. Curr. Med. Chem. 18(25), 3889-3928 (2011).

10 Adjei GO, Kurtzhals JaL, Rodrigues OP et al. Amodiaquine-artesunate vs artemetherlumefantrine for uncomplicated malaria in Ghanaian children: a randomized efficacy and safety trial with one year follow-up. Malaria J. 7(1), 127 (2008).

11 The Rts SCTP. A Phase 3 trial of RTS,S/ AS01 malaria vaccine in African infants. N. Engl. J. Med. 367(24), 2284-2295 (2012).
12 Meunier B, Robert A. Heme as trigger and target for trioxane-containing antimalarial drugs. Acc. Chem. Res. 43(11), 1444-1451 (2010).

13 Haynes RK, Cheu K-W, Li K-Y et al. A partial convergence in action of methylene blue and artemisinins: antagonism with chloroquine, a reversal with verapamil, and an insight into the antimalarial activity of chloroquine. ChemMedChem 6(9), 1603-1615 (2011).

14 Slack RD, Jacobine AM, Posner GH. Antimalarial peroxides: advances in drug discovery and design. Med. Chem. Comm. 3(3), 281-297 (2012).

15 Vennerstrom JL, Arbe-Barnes S, Brun R et al. Identification of an antimalarial synthetic trioxolane drug development candidate. Nature 430(7002), 900-904 (2004).

16 Graham IA, Besser K, Blumer $S$ et al. The genetic map of Artemisia annua L. identifies loci affecting yield of the antimalarial drug artemisinin. Science 327(5963), 328-331 (2010). 


\section{EDITORIAL | Cook}

17 Ro DK, Paradise EM, Ouellet M et al. Production of the antimalarial drug precursor artemisinic acid in engineered yeast. Nature 440(7086), 940-943 (2006).

18 Westfall PJ, Pitera DJ, Lenihan JR et al. Production of amorphadiene in yeast, and its conversion to dihydroartemisinic acid, precursor to the antimalarial agent artemisinin. Proc. Natl Acad. Sci. USA 109(3), E111-E118 (2012).

19 Yadav JS, Thirupathaiah B, Srihari P. A concise stereoselective total synthesis of (+)-artemisinin. Tetrahedron 66(11), 2005-2009 (2010).
20 Zhu C, Cook SP. A concise synthesis of (+)-artemisinin. J. Am. Chem. Soc. 134(33), 13577-13579 (2012).

\section{- Patent}

101 Cook SP, Zhu C: US61484317 (2011). 